J Phys Chem Lett. 2020 January 16; 11(2): 419-426. doi:10.1021/acs.jpclett.9b03489.

\title{
Implementation of Geometry Dependent Charge Flux into Polarizable AMOEBA+ Potential
}

\author{
Chengwen Liu ${ }^{\dagger}$, Jean-Philip Piquemal ${ }^{\dagger, \ddagger, \S}$, Pengyu Ren ${ }^{\dagger}$ \\ tDepartment of Biomedical Engineering, The University of Texas at Austin, Austin, TX 78712, \\ USA \\ ‡Laboratoire de Chimie Théorique, Sorbonne Université, UMR7616 CNRS, Paris, France \\ §Institut Universitaire de France, 75005, Paris, France
}

\begin{abstract}
Molecular dynamics (MD) simulations employing classical force fields (FFs) have been widely used to model molecular systems. The important ingredient of the current FFs, atomic charge, remains fixed during MD simulations despite the atomic environment or local geometry changes. This approximation hinders the transferability of the potential being used in multiple phases. Here we implement a geometry dependent charge flux (GDCF) model into the multipole-based AMOEBA+ polarizable potential. The CF in the current work explicitly depends on the local geometry (bond and angle) of the molecule. To our knowledge, this is the first study that derives energy and force expressions due to GDCF in a multipole-based polarizable FF framework. Due to the inclusion of GDCF, the AMOEBA+ water model is noticeably improved in terms of describing the monomer properties, cluster binding/interaction energy and a variety of liquid properties, including the infrared spectra that previous flexible water models were not able to capture.
\end{abstract}

\section{Graphical Abstract}

Corresponding Author: pren@utexas.edu.

Code Availability

All code is available in the publically accessible TinkerTools Github site as Tinker/AMOEBA+CF and Tinker-OpenMM/AMOEBA + CF branch.

Supporting Information

Additional methodologies, figures, tables, numerical results and references (PDF). 


\section{Geometry Dependent Charge Flux}

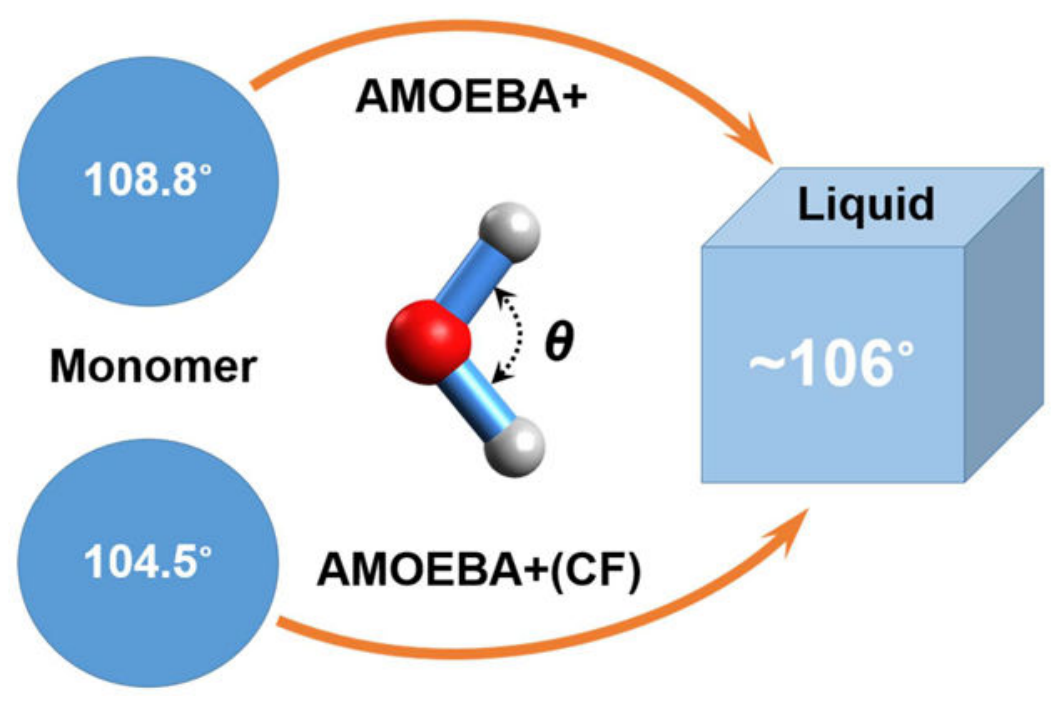

\section{Keywords}

Geometry dependent charges; AMOEBA+ potential; Polarizable water model; Infrared spectra

Classical force fields (FFs) are commonly used to describe inter-and intramolecular interactions in molecular dynamics (MD) simulations. In popular fixed charge FFs, the atomic charges remain fixed during simulations. It is well understood however charges distributions are affected by both chemical environments through polarization effect and local geometry changes. ${ }^{1}$ The former is explicitly treated in "polarizable" FFs such as Drude oscillator, ${ }^{2}$ atomic induced dipole, ${ }^{3}$ and fluctuating charge models, where the charges can be calculated from the energy equilibration, ${ }^{4-6}$ or bond capacity model. ${ }^{7}$ The latter is ignored by almost all classical FFs even though it is well known it causes issues. For example, the $\mathrm{HOH}$ angle of water in gas $(104.5),{ }^{8}$ liquid $(\sim 106)^{9}$ and ice $\left(\sim 109.5^{\circ}\right)^{10}$ cannot be described consistently by common flexible model models. ${ }^{11-12}$ This is due to incorrect dipole derivative of these flexible water models, without accounting for intramolecular charge transfer or charge flux (CF) when water geometry changes. The spectroscopically determined force field (SDFF) electrostatics by Krimm and co-workers was one of the few FFs that adopt a CF contribution. ${ }^{13-15}$ Their study showed that CF is not only the key to the water angle opening from an isolated water molecule to its liquid phase but also helps in describing the conformational potential energy surface of the peptide. ${ }^{13}$ TTM-family models by Xantheas and coworkers are other examples that incorporate $\mathrm{CF}$ effect. ${ }^{16-17}$ Both the SDFF and TTM models show the necessity of incorporating CF for successfully describing vibrational spectroscopy which requires an accurate description of the molecular dipole surface. ${ }^{11,13-17}$ Dinur pointed out that $\mathrm{CF}$ is a first-order contribution to the electrostatic force in general and should not be neglected in MD simulations for flexible molecules. ${ }^{12}$ Dinur and Hagler proposed a geometry dependent charge flux (GDCF) model, where atomic charges are explicitly dependent on the local geometry (bond, angle and torsion), for a series 
of small organic molecules. Based on the molecular dipole moments of organic molecules and amino acids calculated with density functional theory and point-charge FFs, Jensen and coworkers concluded that the majority $(\sim 85 \%)$ of the conformational dependence of molecular dipole moments can be attributed to the pure geometry effect and the remainder should be explicitly modeled by GDCF model. ${ }^{18-19}$ Thus they suggested an inclusion of CF contribution from bond, angle and dihedral for developing more transferable FFs. By contrast, Dinur and Hagler demonstrated that CF due to bonds and angles is much more significant than that from dihedral. ${ }^{20}$

AMOEBA (Atomic Multipole Optimized Energetics for Biomolecular Applications) FF uses multipoles up to quadrupole to describe electrostatics and induced dipole to capture the nonadditive many-body effect. ${ }^{3,21-24}$ AMOEBA+ potential was developed very recently, where the "short-range" physics including charge penetration ${ }^{25}$ and intermolecular charge transfer ${ }^{26}$ effects were incorporated. In addition, in the AMOEBA+ model, the original Thole polarization model (direct component) ${ }^{27-29}$ was improved to better capture the MP2 many-body energy, along with better combining rules for empirical van der Waals potential. ${ }^{30}$ Nevertheless, the atomic charge in the AMOEBA+ model is still independent of the local geometry changes. Consequently, similar to the current AMOEBA model and other flexible water models, an artificially large equilibrium $\mathrm{HOH}$ angle of $108.8^{\circ}$ was used in AMOEBA+ in order to reach the correct bending angle in the liquid phase $\left(\sim 106^{\circ}\right)$. As mentioned above, it has been well recognized that this is attributed to the fact that the molecular charge distribution cannot properly adjust to the changing geometry, i.e. dipole derivatives are incorrect. In this work, we implement the GDCF model into AMOEBA+ potential. Different from the model proposed by Dinur and Hagler, ${ }^{20}$ only the $\mathrm{CF}$ along bond and angle contributions are considered. In addition, we systematically integrated the GDCF model with permanent and polarizable multipole interactions, with analytical gradients. With $\mathrm{CF}$ inclusion, the previous AMOEBA+ water model was reparametrized using ForceBalance toolkit ${ }^{31}$ by targeting on both gas-phase $\mathrm{QM}$ data and liquid-phase observables, resulting in the current AMOEBA+(CF) water model.

To use a water molecule as an illustration (Figure 1), CF along each bond is described as a function of the deviation of bond and angle from their equilibrium values. For water, experimental angle and bond length $\left(104.5^{\circ}\right.$ and $\left.0.9572 \AA\right)$ are used as the reference. The CF direction rules are kept the same as those suggested by Dinur and Hagler. ${ }^{20}$ The CF on each atom is added to its permanent monopole values prior to energy and force computations. Derivation of the GDCF model in the AMOEBA+ framework, including permanent multipole and polarization energy and forces, is detailed in the Methodologies section and Supporting Information (SI). The newly parametrized AMOEBA+(CF) water model is extensively compared with the previous AMOEBA+ and other advanced water models. Below, to clearly demonstrate the improvement due to the inclusion of GDCF, we systematically report the results on the new water model from monomer to clusters and to liquid properties. 


\section{Water monomer properties.}

As mentioned above, GDCF potentially leads to the automatic angle opening in liquid phase simulations of water. This allows us to use an angle of experimental geometry as the initial parameter. As shown in Table 1, AMOEBA+(CF) angle and bond final parameters (after cluster/liquid refinement) resemble the experimental values of an isolated water molecule. The force constants of bonded terms are adjusted slightly to better describe the experimental vibration frequencies of an isolated monomer. In addition, using the minimal-energy geometry, we show that the quality of molecular dipole, quadrupole and polarizability is significantly improved over the AMOEBA+ model, which has a compensative bigger dipole and quadrupole moments but smaller molecular polarizability. Early AMOEBA models are not shown here but they are quite similar to AMOEBA+. As expected, the final/optimal nonbonded parameters change only in small fraction comparing to those of the AMOEBA+ model (Table S1).

\section{Water dimer properties.}

Both the binding energy (Eq. 1) and interaction energy (Eq. 2) were computed. The former used optimized monomers as references where keeps the monomers the same as in the dimer geometry. The dissociation energy (negative of binding energy) of the canonical hydrogenbonding water dimer from the AMOEBA $+(\mathrm{CF})$ model is $4.87 \mathrm{kcal} / \mathrm{mol}$, which is slightly improved comparing to AMOEBA+ $(4.81 \mathrm{kcal} / \mathrm{mol})$ and in agreement with the $\mathrm{CCSD}(\mathrm{T}) / \mathrm{CBS}^{37}$ value of $4.98 \mathrm{kcal} / \mathrm{mol}$. In addition, the AMOEBA+(CF) predicts intermolecular interaction energy components, including electrostatics, induction and van der Waals matching those from the SAPT2+ model (Figure S1). Besides the canonical HB dimer, the "Smith dimers" 38 formed through different directional HBs often serve as model dimers to examine the anisotropy of water models. AMOEBA+(CF) predicts the binding energy of 10 Smith dimers extremely well with an RMSE $0.25 \mathrm{kcal} / \mathrm{mol}$ comparing to that of $0.59 \mathrm{kcal} / \mathrm{mol}$ of AMOEBA+ model. As an additional comparison, the results from the MBUCB water model, ${ }^{39}$ which shares many similarities with the AMOEBA+ model, are also provided in Table 2.

$$
\begin{gathered}
\Delta E_{\text {binding }}=E_{\text {dimer }}^{\text {opt }}-2 E_{\text {monomer }}^{\mathrm{pt}} \\
\Delta E_{\text {interaction }}=E_{\text {dimer }}-E_{\text {monomer } 1}^{*}-E_{\text {monomer } 2}^{*}
\end{gathered}
$$

In the above equations, superscripts opt means optimized geometry and $*$ means monomer geometry kept the same as in dimer.

\section{Larger water clusters.}

We demonstrate here that AMOEBA+(CF) model is capable of accurately predicting both binding energy $(B E)$ and interaction energy (IE) for large water clusters from trimer to 17mers comparing to available $\mathrm{CCSD}(\mathrm{T}) / \mathrm{CBS}$ data. Here the BEs of water models were calculated from FF-optimized geometry while the CCSD(T)/CBS used the MP2-optimized geometry. The IEs were calculated using the MP2-optimized cluster geometry for both the 
QM and FFs. As seen from Figure 2a-c for BEs for clusters from trimer to 17-mers, MB$\mathrm{UCB}$ and AMOEBA+ give overall RMSEs of 2.95 and $1.89 \mathrm{kcal} \cdot \mathrm{mol}^{-1}$ respectively. AMOEBA+(CF) model remarkably reduces the error to $0.67 \mathrm{kcal} / \mathrm{mol}$ (Table S2). For the IEs of tetra-, penta-and hexamer isomers, the AMOEBA+ model gives an RMSE of 1.74 $\mathrm{kcal} / \mathrm{mol}$ and AMOEBA+(CF) significantly reduces the error to $0.36 \mathrm{kcal} / \mathrm{mol}$, which is slightly better than MB-pol water model $(0.39 \mathrm{kcal} / \mathrm{mol})$ (Table S3). It is worth mentioning that the MB-pol model was explicitly fitted on the IEs of water clusters ${ }^{41}$ while only the BEs were included as the targets in AMOEBA+ $(\mathrm{CF})$ parameterization (in addition to selected liquid properties). These results further indicate the importance of a correct monomer geometry for a flexible water model to accurately capture the complicated energy surfaces of water clusters.

\section{Liquid properties.}

As shown above, the addition of CF to AMOEBA+ significantly improves its ability to describe the structural and energetic properties of gas-phase water clusters. Here we examine the performance of AMOEBA $+(\mathrm{CF})$ in liquid. Overall, AMOEBA $+(\mathrm{CF})$ model maintains the quality of the AMOEBA+ model on predicting the average thermodynamic, structural and dynamic properties over a broad range of temperatures (Figure 3). For six thermodynamic properties included in the parametrization targets, density (Figure 3a), enthalpy of vaporization (Figure 3b), thermal expansion coefficient (Figure 3c) and isothermal compressibility (Figure 3e), AMOEBA+(CF) captures the experimental properties as well as the previous AMOEBA+ model. AMOEBA+(CF) liquid water density at room temperature is $997.4 \pm 0.1 \mathrm{~kg} / \mathrm{m}^{3}$, almost exactly the same as experimental measurement $\left(997.0 \mathrm{~kg} \cdot \mathrm{m}^{-3}\right)$, when a larger box of $60 \AA^{3}$ and a van der Waals cutoff of 12 $\AA$ are used. At $298 \mathrm{~K}, \mathrm{AMOEBA}+(\mathrm{CF})$ is slightly worse than the AMOEBA+ model by 1.0 $\mathrm{cal} / \mathrm{mol} / \mathrm{K}$ on predicting the isobaric heat capacity (Figure $3 \mathrm{f}$ ), which is known to be difficult for flexible classical water models due to nuclear quantum effect. ${ }^{42}$ AMOEBA+(CF) notably improves the agreement with experiment for the static dielectric constant in the whole temperature range comparing to AMOEBA+ (Figure 3d). This can be attributed to a better quality of electrostatics, including CF-augmented-multipole moments and polarizability of the AMOEBA+(CF) model than AMOEBA+ (Table 1). At ambient conditions $(298 \mathrm{~K}, 1$ atm), AMOEBA $+(\mathrm{CF})$ results in a static dielectric constant of $78.8 \pm 3.1$, in excellent agreement with the experiment (78.4). The liquid properties which were not included in the parametrization targets are also well reproduced by AMOEBA+(CF) model, as shown in Figure $3 \mathrm{~g}$ and Figure S2 (SI) for the radial distribution function at ambient conditions and Figure $3 \mathrm{~h}$ for the self-diffusion constant at a series of temperatures (also Figure S3 and Table $\mathrm{S} 4)$. The average $\mathrm{O}-\mathrm{H}$ bond length and $\mathrm{H}-\mathrm{O}-\mathrm{H}$ angle in liquid by AMOEBA $+(\mathrm{CF})$ are 0.96 $\AA$ and $105.5^{\circ} \pm 4.7^{\circ}$, while experimental values are $0.97 \AA$ and $106.1^{\circ} \pm 1.8^{\circ} .{ }^{32}$ Coincidently, $\mathrm{AMOEBA}+(\mathrm{CF})$ liquid $\mathrm{HOH}$ angle is consistent with the value of $a b$ initio $\mathrm{MD}$ simulations $\left(105.5^{\circ}\right) .{ }^{43}$ By contrast, AMOEBA+ gives an appropriate average angle $\left(106.3^{\circ} \pm 4.7^{\circ}\right)$ but shorter bond length $(0.95 \AA)$ in liquid (also in cluster as shown by Hughes et al. $\left.{ }^{44}\right)$. It is worth noting that although both water models correctly predict the average angle, as mentioned above, AMOEBA+ angle is originated from an artificially large equilibrium angle parameter $\left(108.8^{\circ}\right)$ while the AMOEBA+(CF) water automatically expanded from $104.5^{\circ}$ in 
isolation to $105.5^{\circ}$ in liquid due to $\mathrm{CF}$. The average amount of charges transferred for a water molecule due to the geometrical deviation is only $-0.0031 e$ on the oxygen atom, of which $-0.0040 e$ is contributed from angle bending, $+0.0004 e$ from symmetrical bond stretching and $+0.0005 e$ from asymmetrical bond stretching.

\section{Infrared spectra of liquid water.}

It has been shown by the SDFF water model that charge flux is necessary to describe the dipole surface and vibrational spectra. ${ }^{15}$ It is also observed that iAMOEBA fails to predict the correct relative intensity of experimental infrared (IR) spectra. ${ }^{45}$ To investigate the impact of CF on the liquid IR spectra, we examined several AMOEBA-based water models either with or without CF. IR spectra were obtained within linear response theory through Fourier transforms of time correlation of net dipole (simulation details in SI). To compare with experimental IR spectra, corrections accounting for quantum effects were added to the calculated IR intensity by using a previously suggested approach. ${ }^{46}$ Figure 4 clearly indicates that without CF implementation, both AMOEBA14 and AMOEBA+ models predict a higher bending peak $\left(\sim 1600 \mathrm{~cm}^{-1}\right)$ than the libration peak $\left(\sim 80 \mathrm{~cm}^{-1}\right)$, which was similarly observed for the AMOEBA03 water (Figure S4) and iAMOEBA models. ${ }^{45}$ By contrast, AMOEBA $+(\mathrm{CF})$ reduces the height of the bending peak and results in correct relative intensity comparing to the experiment. In the $\mathrm{OH}$ stretching region, it is seen that the stretching is shifted to low frequencies (blue shift) from $3755 \mathrm{~cm}^{-1}$ (asymmetric) and 3656 $\mathrm{cm}^{-1}$ (symmetric) of an isolated water molecule (Table 1) to $\sim 3550 \mathrm{~cm}^{-1}$ (Figure S4). This blue shift magnitude is insufficient comparing to experiment, which can be attributed to the lack of the explicit treatment of the nuclear quantum effect in this high-frequency region for classical models. ${ }^{47-48}$ With an adjusted bond stretching force constant, AMOEBA+(CF) model is able to predict the correct peak position $\left(\sim 3400 \mathrm{~cm}^{-1}\right)$. Additionally, it is clear that a "stiffer" bond stretching force constant also helps to prevent the peak splitting observed in AMOEBA+(CF) using gas-phase force constant, as well as other models (Figure 4 and Figure S4). Thus this modified model is suggested for vibrational spectroscopy simulation in water. This modification on the bond stretching force constant has no effect on average liquid thermodynamic or dynamic properties we have computed.

\section{Ice properties.}

Three crystal forms of ice, Ih, Ic and II, were simulated at $1 \mathrm{~atm}$ pressure and experimental temperatures by employing AMOEBA+ and AMOEBA+(CF) water models. The computational details are provided in SI and the results are summarized in Table S11 and S12. The average density simulated by our models agree reasonably well with experiment within $\sim 2 \%$ for Ih, $\sim 4 \%$ for Ic and $\sim 6 \%$ for ice II (Table S11), which can be attributed to two factors: (1) the lack of nuclear quantum effect, which normally leads to a reduction of the simulated density and (2) the exclusion of solid-phase properties in our parametrization. As shown in Table S12, AMOEBA+(CF) model shows the reasonable capability to predict other properties of ice Ih without explicitly parametrizing to ice data. For example, simulated enthalpy of sublimation for ice Ih at $269 \mathrm{~K}$ and $1 \mathrm{~atm}$ is $-12.13 \mathrm{kcal} / \mathrm{mol}$, which excellently agrees with experiment $(-12.20 \mathrm{kcal} / \mathrm{mol}) .{ }^{50} \mathrm{By}$ contrast, TTM-family models over-predict enthalpy of sublimation of ice Ih, with TTM4-F being $-14.40 \mathrm{kcal} / \mathrm{mol}$ and TTM2-F being 
$13.39 \mathrm{kcal} / \mathrm{mol}^{51}$ The average intermolecular OO distance $(2.73 \AA)$ is $\sim 1 \%$ shorter than experimental value $(2.76 \AA)$, which is consistent with slightly higher simulated density. The intramolecular $\mathrm{OH}$ distance $(0.97 \AA)$ is in agreement with experiment $(0.98 \AA) .52$

Experimental value of the $\mathrm{HOH}$ angle is commonly referenced as ideal tetrahedral angle of 109.5 . A trend of $\mathrm{HOH}$ angle expansion (gas < liquid < ice) consistent to experiment is observed for the AMOEBA+(CF) model in three phases while the AMOEBA+ model gives the opposite trend as gas > liquid > ice.

In summary, we implemented the GDCF model into the multipole-based polarizable AMOEBA+ potential. The GDCF model was originally proposed by Dinur and Hagler and examined on small organic molecules within the point charge electrostatic framework. ${ }^{20} \mathrm{In}$ this work, we integrated the GDCF model with atomic multipole electrostatics (with permanent multipoles up to quadrupole) and many-body atomic dipole polarization in the AMOEBA+ framework. The energy and force expressions due to the inclusion of GDCF were derived. We examined the impact of GDCF by updating the AMOEBA+ water model. Our results indicate that GDCF allows the use of appropriate equilibrium angle and bond length for an isolated water molecule which will spontaneously adjust to the correct values in liquid. The correct monomer geometry and GDCF lead to noticeable improvements in both the binding energy and the interaction energy of water clusters. Finally, AMOEBA+ (CF) water model shows excellent liquid properties, along with improved IR spectra in terms of capturing the relative intensity of bending and libration peaks.

The preliminary implementation of AMOEBA+(CF) was finished on our developing version of Tinker (CPU) and Tinker-OpenMM (GPU). MD simulations under NVE ensemble were carried out on both CPU and GPU codes to evaluate the energy conservation of the AMOEBA+(CF) model (simulation details in SI). To take advantage of the double precision of Tinker CPU code, simulation using a very tight induced dipole convergence (polar-eps $10^{-12} \mathrm{D}$ ) with the iterative SCF method leads to only $-0.01 \mathrm{kcal} / \mathrm{mol} / \mathrm{ns}$ of total energy drift (out of $\sim-3800 \mathrm{kcal} / \mathrm{mol}$ ) (Figure S5a). Alternatively, simulation with the "OPT4" extrapolated polarization scheme ${ }^{53}$ on CPU gives a total energy drift of $-0.03 \mathrm{kcal} / \mathrm{mol} / \mathrm{ns}$ (Figure S5b). As expected, simulations on Tinker-OpenMM GPU (mixed precision) give a greater total energy drift of $-0.2 \mathrm{kcal} / \mathrm{mol} / \mathrm{ns}$ (Figure S5c) and $+0.1 \mathrm{kcal} / \mathrm{mol} / \mathrm{ns}$ (Figure S5d) for SCF (polar-eps $10^{-6} \mathrm{D}$ due to single precision) and "OPT4" scheme respectively. These results indicate the excellent energy conservation of our implementation. Besides, it is shown that a negligible additional cost (less than 1\%) arises from the GDCF algorithms. Further code implementation and optimization in the latest version of Tinker, ${ }^{54}$ TinkerOpenMM ${ }^{55}$ and Tinker-HP ${ }^{56}$ are ongoing, as well as the parametrization of AMOEBA+(CF) for a wide range of molecular systems.

\section{METHODOLOGIES}

Here we briefly describe the theoretical methodologies of CF implementation. AMOEBA+ potential adopts atomic multipoles to represent atomic charge distributions, with short-range penetration effect. ${ }^{25}$ Multipole moments on atom $i$ can be expressed as 


$$
M_{i}=\left[q, \mu_{x}, \mu_{y}, \mu_{z}, \Theta_{x x}, \ldots\right]
$$

To implement the GDCF model, we followed the algorithms proposed by Dinur and Hagler ${ }^{20}$ by only including the bond and angle contributions. For a pair of bonded atoms $a$ and $b$, CFs on atom $a$ and $b$ due to bond stretching are expressed as

$$
d q_{a}=-d q_{b}=j_{b}\left(r_{a b}-r_{a b}^{0}\right)
$$

where $r_{a b}$ and $r_{a b}^{0}$ are the actual and equilibrium bond lengths; $j_{b}$ is the determining parameter specific to this bond. The CF direction rule is kept the same as previous work. ${ }^{20}$ Briefly, $-d q$ is added to the (1) atom with a bigger atomic number; (2) atom with more connections if rule (1) is not applicable; (3) atom with more connected hydrogen atoms if both (1) and (2) are not applicable. For an angle $\angle a b c$, the CFs due to angle bending are expressed as

$$
\begin{gathered}
d q_{a}=j_{\theta 1}\left(\theta-\theta_{0}\right)+j_{b 1}^{\prime}\left(r_{b c}-r_{b c}^{0}\right) \\
d q_{c}=j_{\theta 2}\left(\theta-\theta_{0}\right)+j_{b 2}^{\prime}\left(r_{a b}-r_{a b}^{0}\right) \\
d q_{b}=-\left(d q_{a}+d q_{c}\right)
\end{gathered}
$$

where $\theta$ and $\theta_{0}$ are actual and equilibrium angle values; the second terms on the right side is due to asymmetric stretching, in which case change of $r_{b c}$ also affects atom $a . j_{\theta 1}, j_{\theta 2}, j_{b 1}^{\prime}$ and $j_{b 2}^{\prime}$ are CF parameters determined by the chemistry (atom types). The initial CF parameters of water were derived by fitting to the molecular dipole surface using MP2/augcc-pvtz level of theory. In ForceBalance optimization, only the $j_{\theta}$ is allowed to be further optimized as we found it is more sensitive to the $\mathrm{HOH}$ angle than the bond-related $\mathrm{CF}$ parameters. For organic molecules and peptides, density functional theory may be applied to calculate the dipole surface, as also suggested by other researchers. ${ }^{18-19}$

With the $\mathrm{CF} d q_{i}$ for atomic site $i$ determined for a given geometry, the monopole in AMOEBA + multipole moments then is replaced by $q+d q_{i}$. The modified multipole is then

$$
M_{i}^{\prime}=\left[q+d q_{i}, \mu_{x}, \mu_{y}, \mu_{z}, \Theta_{x x}, \ldots\right]
$$

With the above multipoles, permanent electrostatics of AMOEBA+ is calculated as

$$
E_{\text {ele }}=\sum_{i, j} M_{i}^{\prime} T^{\text {damped }} M_{j}^{\prime}
$$

wherein the AMOEBA+ potential, the multipole-multipole interaction $T$ matrix is damped to account for the charge penetration effect. ${ }^{25-26}$ Induced dipole and polarization energy are 
calculated in the same manner as current AMOEBA+ model with the updated multipoles $M^{\prime}$.

In order to use the GDCF model in MD simulations, one needs the gradient of the potential energy w.r.t. atom coordinate. We found that the final form of the electrostatic and polarization forces can be expressed in the following formula

$$
F_{i, \alpha}(C F)=F_{i, \alpha}+F_{i, \alpha}^{\prime} \quad(\alpha=x, y, z)
$$

The first term on the right side stands for the usual AMOEBA+ electrostatics and polarization terms with $\mathrm{CF}$-updated charges. The second chain-rule term arises from $\mathrm{CF}$ which explicitly depends on the internal bonds and angles. In SI, we show that $F$ ' can be calculated using the accumulated potential on each atom $\left(V_{i}\right)$ and the derivative of CF w.r.t. coordinates $\left(\frac{\partial d i}{\partial \alpha}\right)$. The $V_{i}$ term in AMOEBA+ framework is contributed from permanent multipoles (charge, dipole, and quadrupole) and induced dipole. These expressions of $V_{i}$ are already calculated in the AMOEBA+ potential. $^{25-26}$ In addition, since charges in our model now depends on the atomic coordinates, extra force contribution to Ewald self-energy appears (see detailed derivation in SI).

\section{Supplementary Material}

Refer to Web version on PubMed Central for supplementary material.

\section{ACKNOWLEDGMENT}

The authors are grateful for support by the Robert A. Welch Foundation (F-1691), National Institutes of Health (R01GM106137 and R01GM114237), the Cancer Prevention and Research Institute of Texas (RP160657), National Science Foundation (CHE-1856173) and European Research Council (ERC) under the European Union's Horizon 2020 research and innovation program (grant agreement No 810367), project EMC2.

\section{REFERENCES}

1. Hagler AT, Force field development phase II: Relaxation of physics-based criteria... or inclusion of more rigorous physics into the representation of molecular energetics. J. Comput. Aided Mol. Des 2019, 33 (2), 205. [PubMed: 30506159]

2. Lemkul JA; Huang J; Roux B; MacKerell AD Jr., An Empirical Polarizable Force Field Based on the Classical Drude Oscillator Model: Development History and Recent Applications. Chem Rev 2016, 116 (9), 4983. [PubMed: 26815602]

3. Jing Z; Liu C; Cheng SY; Qi R; Walker BD; Piquemal J-P; Ren P, Polarizable Force Fields for Biomolecular Simulations: Recent Advances and Applications. Annu. Rev. Biophys 2019.

4. Patel S; Brooks CL, Fluctuating charge force fields: recent developments and applications from small molecules to macromolecular biological systems. Molecular Simulation 2006, 32 (3-4), 231.

5. Patel S; Brooks III CL, CHARMM fluctuating charge force field for proteins: I parameterization and application to bulk organic liquid simulations. J Comput Chem 2004, 25 (1), 1. [PubMed: 14634989]

6. Patel S; Mackerell AD Jr.; Brooks III CL, CHARMM fluctuating charge force field for proteins: II Protein/solvent properties from molecular dynamics simulations using a nonadditive electrostatic model. J Comput Chem 2004, 25 (12), 1504. [PubMed: 15224394]

7. Poier PP; Jensen F, Describing Molecular Polarizability by a Bond Capacity Model. J. Chem. Theory Comput 2019, 15 (5), 3093. [PubMed: 30920212] 
8. Benedict WS; Gailar N; Plyler EK, Rotation-Vibration Spectra of Deuterated Water Vapor. J. Chem. Phys 1956, 24 (6), 1139.

9. Ichikawa K; Kameda Y; Yamaguchi T; Wakita H; Misawa M, Neutron-diffraction investigation of the intramolecular structure of a water molecule in the liquid phase at high temperatures. Mol. Phys 1991, 73 (1), 79.

10. Kuhs WF; Lehmann MS, The structure of the ice Ih by neutron diffraction. J. Phys. Chem 1983, 87 (21), 4312.

11. Fanourgakis GS; Xantheas SS, The bend angle of water in ice Ih and liquid water: The significance of implementing the nonlinear monomer dipole moment surface in classical interaction potentials. J. Chem. Phys 2006, 124 (17), 174504. [PubMed: 16689580]

12. Dinur U, "Flexible" water molecules in external electrostatic potentials. J. Phys. Chem 1990, 94 (15), 5669.

13. Palmo K; Mannfors B; Mirkin NG; Krimm S, Inclusion of charge and polarizability fluxes provides needed physical accuracy in molecular mechanics force fields. Chem Phys Lett 2006, 429 (4), 628.

14. Palmo K; Mannfors B; Krimm S, Balanced charge treatment of intramolecular electrostatic interactions in molecular mechanics energy functions. Chem Phys Lett 2003, 369 (3), 367.

15. Mannfors B; Palmo K; Krimm S, Spectroscopically Determined Force Field for Water Dimer: Physically Enhanced Treatment of Hydrogen Bonding in Molecular Mechanics Energy Functions. J Phys Chem A 2008, 112 (49), 12667. [PubMed: 19012387]

16. Burnham CJ; Xantheas SS, Development of transferable interaction models for water. IV. A flexible, all-atom polarizable potential (TTM2-F) based on geometry dependent charges derived from an ab initio monomer dipole moment surface. J. Chem. Phys 2002, 116 (12), 5115.

17. Fanourgakis GS; Xantheas SS, Development of transferable interaction potentials for water. V. Extension of the flexible, polarizable, Thole-type model potential (TTM3-F, v. 3.0) to describe the vibrational spectra of water clusters and liquid water. J. Chem. Phys 2008, 128 (7), 074506. [PubMed: 18298156]

18. Sedghamiz E; Nagy B; Jensen F, Probing the Importance of Charge Flux in Force Field Modeling. J. Chem. Theory Comput 2017, 13 (8), 3715. [PubMed: 28635293]

19. Sedghamiz E; Ghalami F, Evaluating the Effects of Geometry and Charge Flux in Force Field Modeling. J Phys Chem A 2018, 122 (19), 4647. [PubMed: 29698601]

20. Dinur U; Hagler AT, Geometry-dependent atomic charges: Methodology and application to alkanes, aldehydes, ketones, and amides. J Comput Chem 1995, 16 (2), 154.

21. Ren PY; Ponder JW, Polarizable atomic multipole water model for molecular mechanics simulation. J. Phys. Chem. B 2003, 107 (24), 5933.

22. Shi Y; Xia Z; Zhang J; Best R; Wu C; Ponder JW; Ren P, Polarizable Atomic Multipole-Based AMOEBA Force Field for Proteins. J. Chem. Theory Comput 2013, 9 (9), 4046. [PubMed: 24163642]

23. Zhang C; Bell D; Harger M; Ren P, Polarizable Multipole-Based Force Field for Aromatic Molecules and Nucleobases. J. Chem. Theory Comput 2017, 13 (2), 666. [PubMed: 28030769]

24. Ponder JW; Wu C; Ren P; Pande VS; Chodera JD; Schnieders MJ; Haque I; Mobley DL; Lambrecht DS; DiStasio RA Jr.; Head-Gordon M; Clark GN; Johnson ME; Head-Gordon T, Current status of the AMOEBA polarizable force field. J Phys Chem B 2010, 114 (8), 2549. [PubMed: 20136072]

25. Rackers JA; Wang Q; Liu C; Piquemal J-P; Ren P; Ponder JW, An optimized charge penetration model for use with the AMOEBA force field. Phys. Chem. Chem. Phys 2017, 19 (1), 276.

26. Liu C; Piquemal J-P; Ren P, AMOEBA+ Classical Potential for Modeling Molecular Interactions. J. Chem. Theory Comput 2019, 15 (7), 4122. [PubMed: 31136175]

27. Liu C; Qi R; Wang Q; Piquemal JP; Ren P, Capturing Many-Body Interactions with Classical Dipole Induction Models. J Chem Theory Comput 2017, 13 (6), 2751. [PubMed: 28482664]

28. Thole BT, Molecular Polarizabilities Calculated with a Modified Dipole Interaction. Chem Phys 1981, 59 (3), 341.

29. van Duijnen PT; Swart M, Molecular and Atomic Polarizabilities: Thole's Model Revisited. J Phys Chem A 1998, 102 (14), 2399. 
30. Qi R; Wang Q; Ren P, General van der Waals potential for common organic molecules. Bioorg Med Chem 2016, 24 (20), 4911. [PubMed: 27519463]

31. Wang LP; Martinez TJ; Pande VS, Building Force Fields: An Automatic, Systematic, and Reproducible Approach. J Phys Chem Lett 2014, 5 (11), 1885. [PubMed: 26273869]

32. Ben-Naim A, Molecular Theory of Water and Aqueous Solutions. World Scientific: 2011.

33. Clough SA; Beers Y; Klein GP; Rothman LS, Dipole moment of water from Stark measurements of H2O, HDO, and D2O. J. Chem. Phys 1973, 59 (5), 2254.

34. Verhoeven J; Dymanus A, Magnetic Properties and Molecular Quadrupole Tensor of the Water Molecule by Beam-Maser Zeeman Spectroscopy. J. Chem. Phys 1970, 52 (6), 3222.

35. Murphy WF, The Rayleigh depolarization ratio and rotational Raman spectrum of water vapor and the polarizability components for the water molecule. J. Chem. Phys 1977, 67 (12), 5877.

36. Maroulis G, Hyperpolarizability of $\mathrm{H} 2 \mathrm{O}$ revisited: accurate estimate of the basis set limit and the size of electron correlation effects. Chem Phys Lett 1998, 289 (3-4), 403.

37. Tschumper GS; Leininger ML; Hoffman BC; Valeev EF; Schaefer HF; Quack M, Anchoring the water dimer potential energy surface with explicitly correlated computations and focal point analyses. J. Chem. Phys 2002, 116 (2), 690.

38. Smith BJ; Swanton DJ; Pople JA; III HFS; Radom L, Transition structures for the interchange of hydrogen atoms within the water dimer. J. Chem. Phys 1990, 92 (2), 1240.

39. Das AK; Urban L; Leven I; Loipersberger M; Aldossary A; Head-Gordon M; Head-Gordon T, Development of an Advanced Force Field for Water Using Variational Energy Decomposition Analysis. J. Chem. Theory Comput 2019, 15 (9), 5001. [PubMed: 31408601]

40. Laury ML; Wang LP; Pande VS; Head-Gordon T; Ponder JW, Revised Parameters for the AMOEBA Polarizable Atomic Multipole Water Model. J Phys Chem B 2015, 119 (29), 9423. [PubMed: 25683601]

41. Reddy SK; Straight SC; Bajaj P; Pham CH; Riera M; Moberg DR; Morales MA; Knight C; Götz AW; Paesani F, On the accuracy of the MB-pol many-body potential for water: Interaction energies, vibrational frequencies, and classical thermodynamic and dynamical properties from clusters to liquid water and ice. J. Chem. Phys 2016, 145 (19), 194504. [PubMed: 27875875]

42. Vega C; Conde MM; McBride C; Abascal JLF; Noya EG; Ramirez R; Sesé LM, Heat capacity of water: A signature of nuclear quantum effects. J. Chem. Phys 2010, 132 (4), 046101. [PubMed: 20113070]

43. Silvestrelli PL; Parrinello M, Structural, electronic, and bonding properties of liquid water from first principles. J. Chem. Phys 1999, 111 (8), 3572.

44. Hughes ZE; Ren E; Thacker JCR; Symons BCB; Silva AF; Popelier PLA, A FFLUX Water Model: Flexible, Polarizable and with a Multipolar Description of Electrostatics. J Comput Chem 2019, doi: $10.1002 /$ jcc.26111.

45. Wang LP; Head-Gordon T; Ponder JW; Ren P; Chodera JD; Eastman PK; Martinez TJ; Pande VS, Systematic improvement of a classical molecular model of water. J Phys Chem B 2013, 117 (34), 9956. [PubMed: 23750713]

46. Gaigeot M-P; Sprik M, Ab Initio Molecular Dynamics Computation of the Infrared Spectrum of Aqueous Uracil. J. Phys. Chem. B 2003, 107 (38), 10344.

47. Medders GR; Paesani F, Infrared and Raman Spectroscopy of Liquid Water through "FirstPrinciples” Many-Body Molecular Dynamics. J. Chem. Theory Comput 2015, 11 (3), 1145. [PubMed: 26579763]

48. Kapil V; VandeVondele J; Ceriotti M, Accurate molecular dynamics and nuclear quantum effects at low cost by multiple steps in real and imaginary time: Using density functional theory to accelerate wavefunction methods. J. Chem. Phys 2016, 144 (5), 054111. [PubMed: 26851912]

49. Maréchal Y, The molecular structure of liquid water delivered by absorption spectroscopy in the whole IR region completed with thermodynamics data. J. Mol. Struct 2011, 1004 (1), 146.

50. Murphy DM; Koop T, Review of the vapour pressures of ice and supercooled water for atmospheric applications. Quarterly Journal of the Royal Meteorological Society 2005, 131 (608), 1539.

51. Burnham CJ; Anick DJ; Mankoo PK; Reiter GF, The vibrational proton potential in bulk liquid water and ice. J. Chem. Phys 2008, 128 (15), 154519. [PubMed: 18433247] 
52. Floriano MA; Klug DD; Whalley E; Svensson EC; Sears VF; Hallman ED, Direct determination of the intramolecular O-D distance in ice Ih and Ic by neutron diffraction. Nature 1987, 329 (6142), 821.

53. Simmonett AC; IV FCP; Ponder JW; Brooks BR, An empirical extrapolation scheme for efficient treatment of induced dipoles. J. Chem. Phys 2016, 145 (16), 164101. [PubMed: 27802661]

54. Rackers JA; Wang Z; Lu C; Laury ML; Lagardère L; Schnieders MJ; Piquemal JP; Ren P; Ponder JW, Tinker 8: Software Tools for Molecular Design. J Chem Theory Comput 2018, 14 (10), 5273. [PubMed: 30176213]

55. Eastman P; Swails J; Chodera JD; McGibbon RT; Zhao Y; Beauchamp KA; Wang LP; Simmonett AC; Harrigan MP; Stern CD; Wiewiora RP; Brooks BR; Pande VS, OpenMM 7: Rapid development of high performance algorithms for molecular dynamics. PLoS Comput Biol 2017, 13 (7), e1005659. [PubMed: 28746339]

56. Lagardère L; Jolly LH; Lipparini F; Aviat F; Stamm B; Jing ZF; Harger M; Torabifard H; Cisneros GA; Schnieders MJ; Gresh N; Maday Y; Ren PY; Ponder JW; Piquemal JP, Tinker-HP: a massively parallel molecular dynamics package for multiscale simulations of large complex systems with advanced point dipole polarizable force fields. Chem Sci 2018, 9 (4), 956. [PubMed: 29732110] 


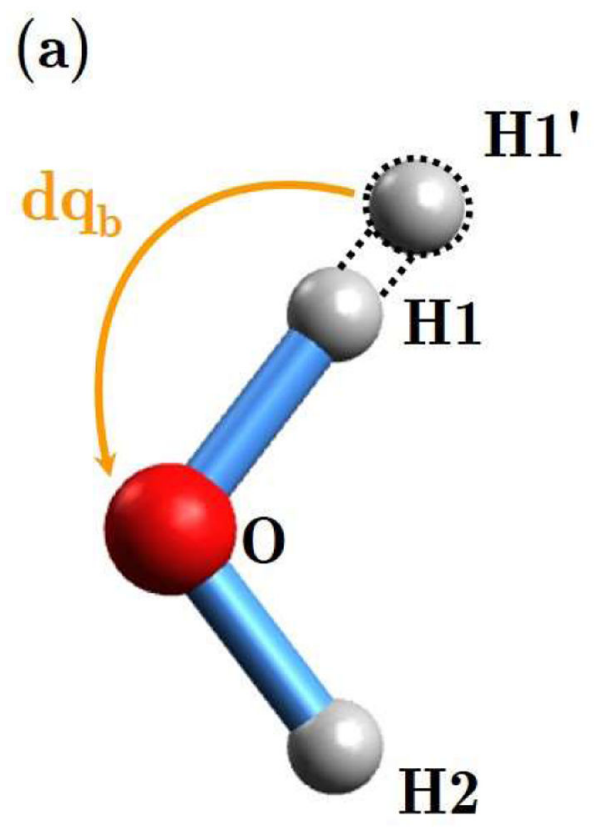

(b)

(a)

$1^{\prime}$

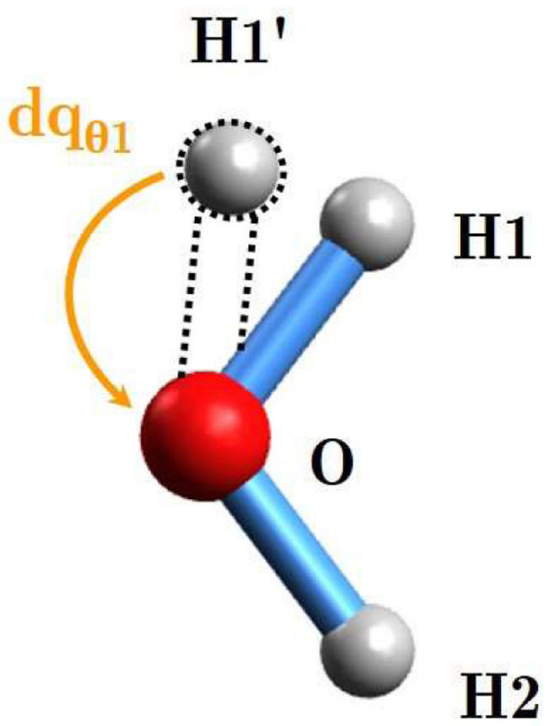

Figure 1.

Schematic illustration of the GDCF model. (a) CF due to bond stretching and (b) CF due to angle bending, where $d q_{b}$ and $d q_{\theta 1}$ represent the absolute charge fluxes due to bond stretching and angle bending. The direction (sign) of CF is defined in the Methodologies section. 
(a) $\left(\mathrm{H}_{2} \mathrm{O}\right)_{3,4,5}$

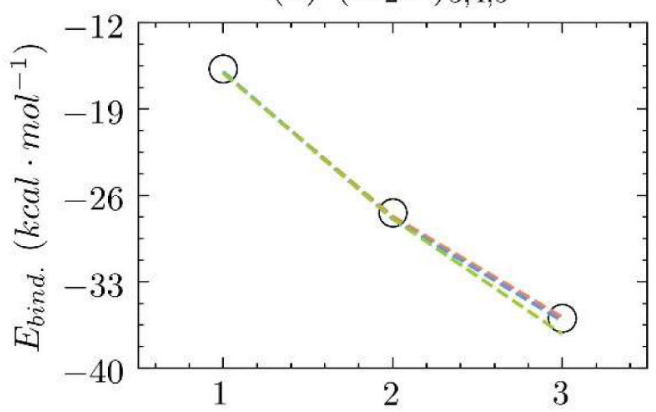

(b) $\left(\mathrm{H}_{2} \mathrm{O}\right)_{6}$

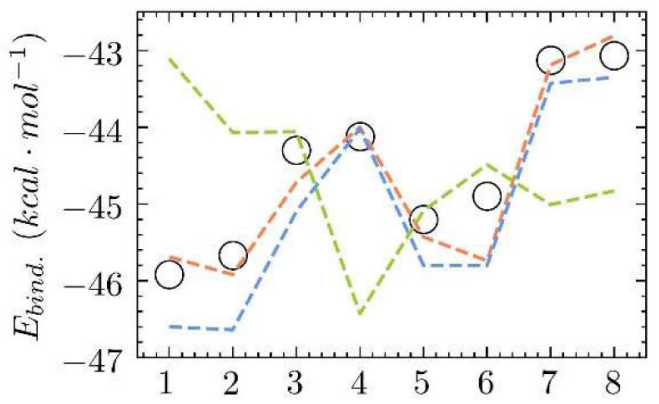

(c) $\left(\mathrm{H}_{2} \mathrm{O}\right)_{16,17}$

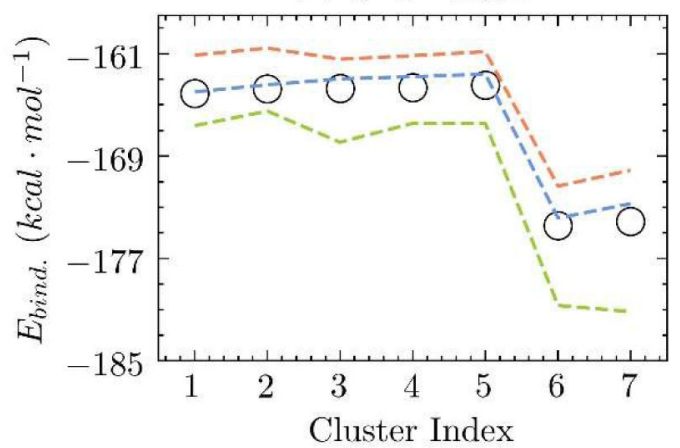

O $\operatorname{CCSD}(\mathrm{T}) / \mathrm{CBS} \quad---\mathrm{AMOEBA}+(\mathrm{CF})$ --- AMOEBA+ (d) $\left(\mathrm{H}_{2} \mathrm{O}\right)_{4}$

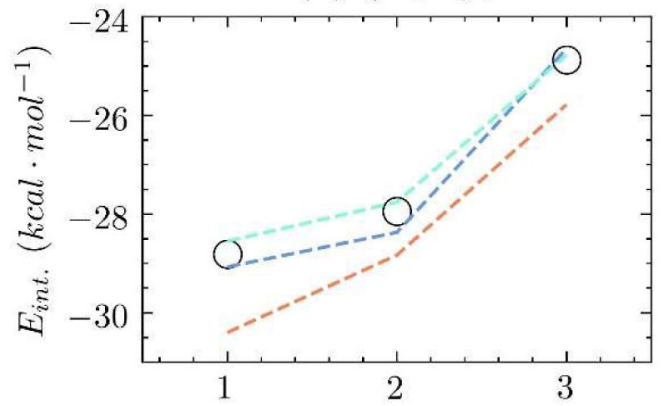

(e) $\left(\mathrm{H}_{2} \mathrm{O}\right)_{5}$

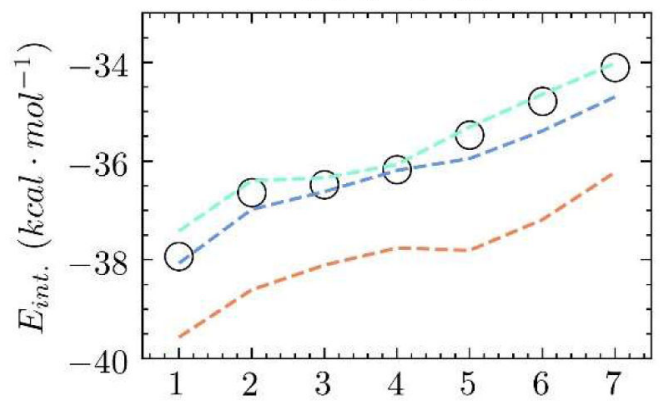

(f) $\left(\mathrm{H}_{2} \mathrm{O}\right)_{6}$

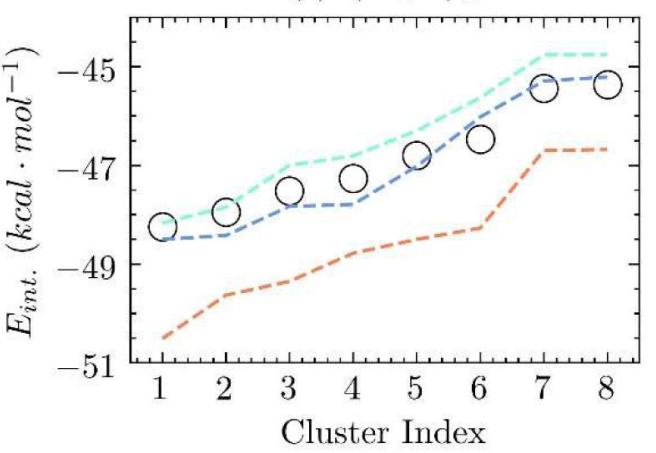

MB-pol

Figure 2.

Binding energy and interaction energy computed with water models compared with available ab initio CCSD(T)/CBS data. (a)-(c): BEs of water trimer to 17-mers and (d)-(f): IEs of water tetramer, pentamer and hexamer conformers. The BEs from MB-UCB ${ }^{39}$ and IEs from MB-pol ${ }^{41}$ water models are provided for comparison. The numerical data, cluster indices and references are provided in Table S2 and Table S3. 
(a) Density

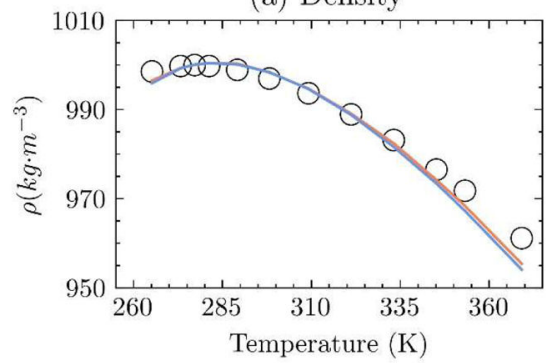

(c) Thermal expansion coefficient

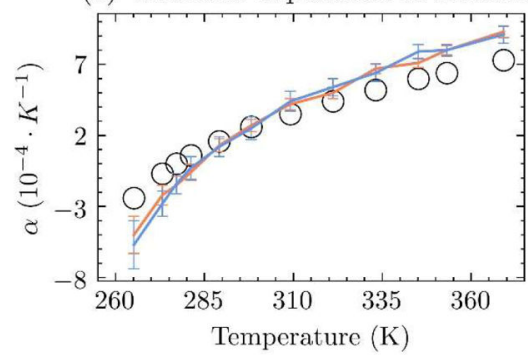

(e) Isothermal compressibility

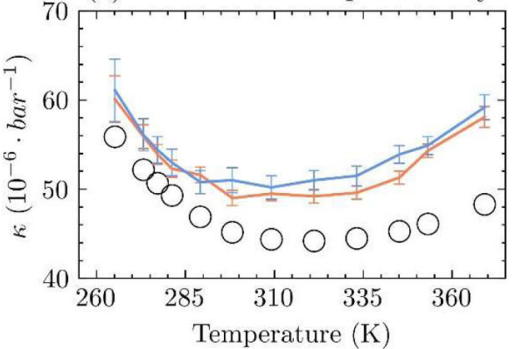

(g) Radial distribution function

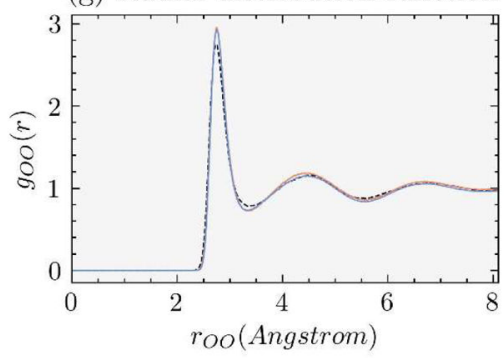

-- Expt

O Expt.

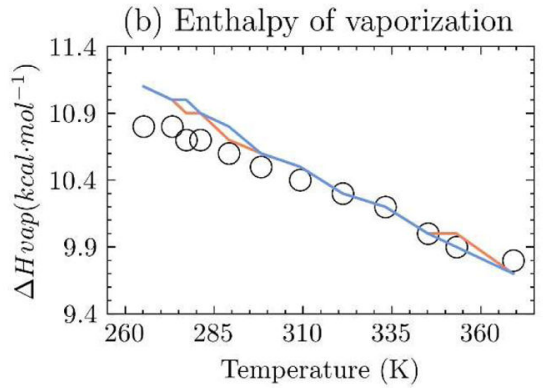

(d) Static dielectric constant
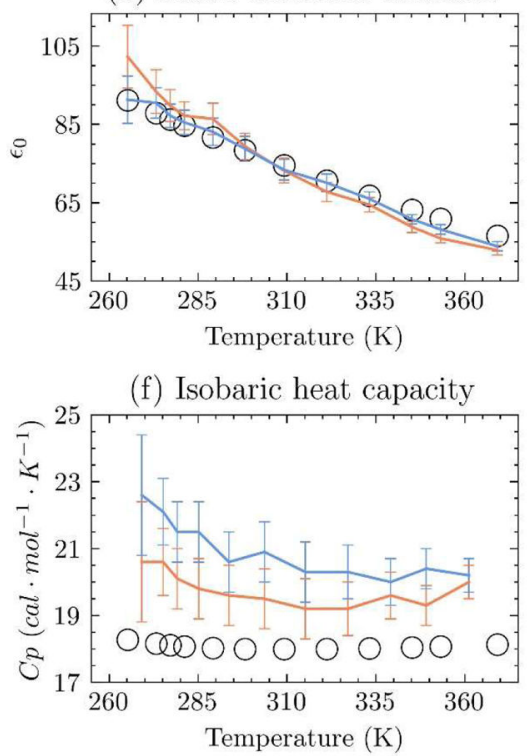

(h) Self-diffusion constant

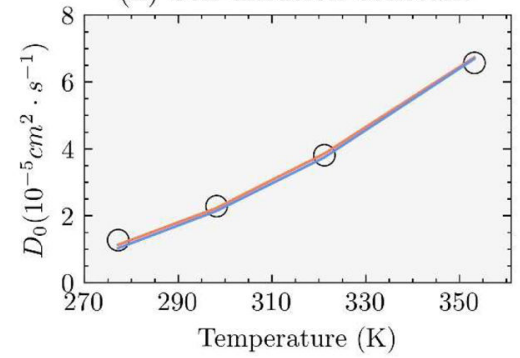

AMOEBA+

AMOEBA $+(\mathrm{CF})$

Figure 3.

Liquid properties of water at a broad range of temperatures and 1 atm pressure. Properties in (a)-(f) were included in the ForceBalance optimization while (g)-(h) were not used in para metrization. Error bars are also plotted if they are notable. The radial distribution function of oxygen-hydrogen and hydrogen-hydrogen pairs are provided in Figure S2. 


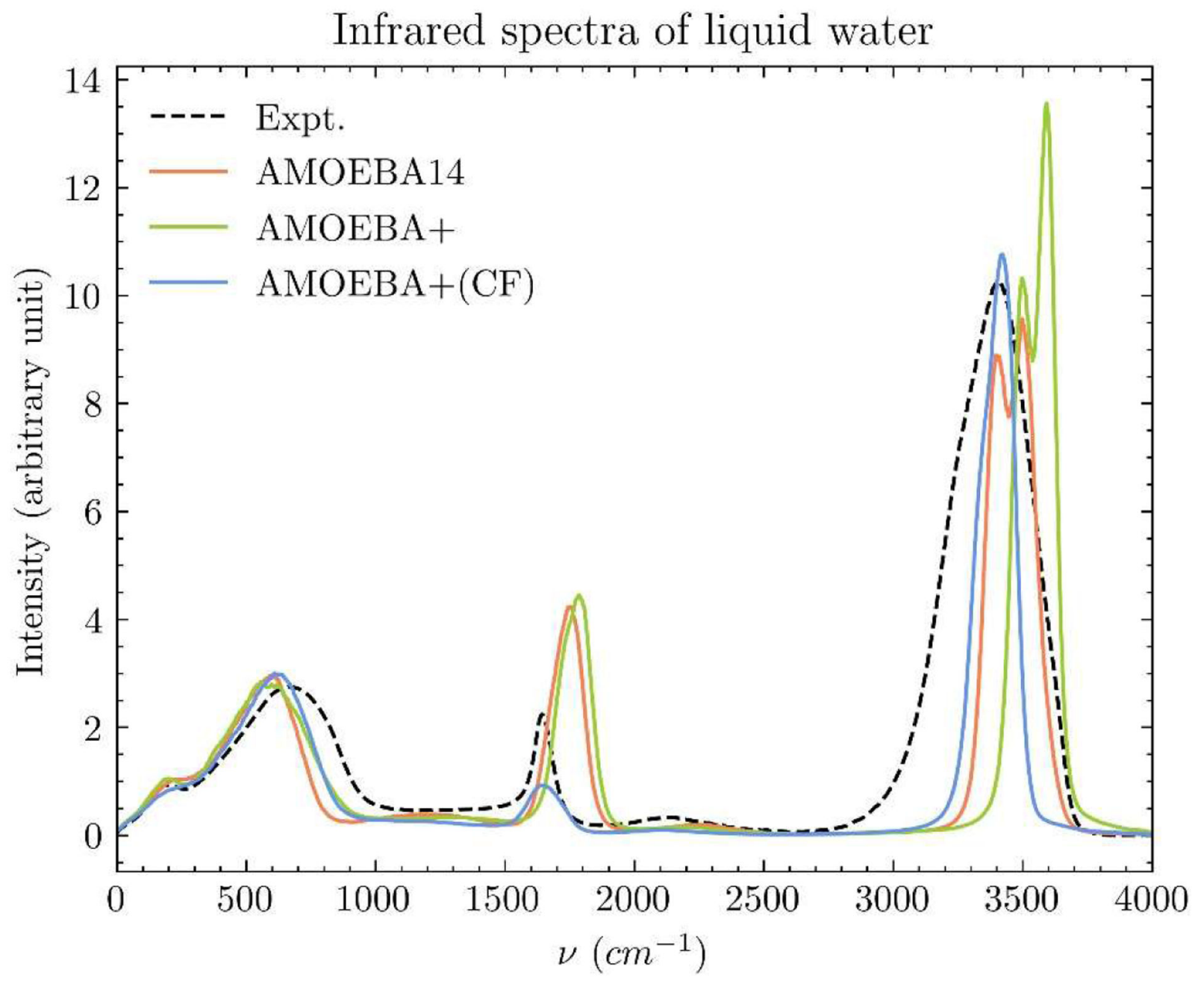

Figure 4.

IR spectra of liquid water calculated with water models with/without charge flux and compared to the experiment. Experimental data were taken from the literature of ambient conditions ( $298 \mathrm{~K}$ and $1 \mathrm{~atm}$ ). ${ }^{49}$ Quantum corrections were added to each calculated spectra using the same approach suggested by a previous study. ${ }^{46}$ More simulation details are provided in SI. 
Table 1.

The vibrational frequencies, geometrical, and moment properties of an isolated water molecule. ${ }^{a}$

\begin{tabular}{ccccc}
\hline Property & & Experiment $^{\boldsymbol{c}}$ & AMOEBA+ $^{\boldsymbol{d}}$ & AMOEB A+(CF) \\
\hline vibrational frequency $^{\boldsymbol{e}}\left(\mathrm{cm}^{-1}\right)$ & $V_{s s}$ & 3657 & $\mathbf{3 6 5 8}$ & $\mathbf{3 6 5 6}$ \\
& $V_{a s}$ & 3756 & 3757 & $\mathbf{3 7 5 5}$ \\
& $V_{b}$ & 1595 & 1627 & $\mathbf{1 5 9 4}$ \\
geometry & $b_{\mathrm{OH}}(\AA)$ & 0.957 & 0.939 & $\mathbf{0 . 9 5 0}$ \\
& $\theta_{\mathrm{HOH}}\left({ }^{\circ}\right)$ & 104.52 & 108.82 & $\mathbf{1 0 4 . 5 4}$ \\
dipole (Debye) & $d_{z}$ & $1.86(1.84)$ & 1.95 & $\mathbf{1 . 8 8}$ \\
Quadrupole (Debye. $\AA)$ & $Q_{x x}$ & $2.63(2.57)$ & 3.17 & $\mathbf{2 . 8 3}$ \\
& $Q_{y y}$ & $-2.50(-2.42)$ & -2.69 & $\mathbf{- 2 . 3 4}$ \\
& $Q_{z z}$ & $-0.13(-0.14)$ & -0.48 & -0.49 \\
Polarizability $(\AA 3)$ & $a_{x x}$ & $1.53(1.47)$ & 1.59 & 1.62 \\
& $a_{y y}$ & $1.42(1.38)$ & 1.21 & $\mathbf{1 . 2 4}$ \\
& $a_{z z}$ & $1.47(1.42)$ & 1.33 & $\mathbf{1 . 3 6}$ \\
\hline
\end{tabular}

avalues in bold indicate a better consistency with the experimental data;

b

$b_{V_{S S}:}$ symmetrical stretching; $v_{S S}$ : asymmetrical stretching; $v b$ : bending vibration;

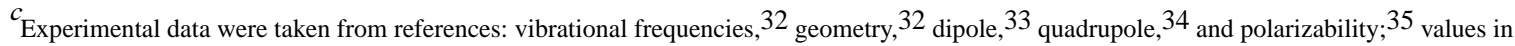
parentheses are ab initio data taken from reference, ${ }^{36}$

${ }^{d}$ Calculated with AMOEBA+ optimized monomer geometry;

${ }^{e}$ Calculated with AMOEBA+(CF) optimized monomer geometry. 
Table 2.

Binding energies (with MP2-optimized geometry) of Smith dimers predicted by several water models comparing to $\operatorname{CCSD}(\mathrm{T}) / \mathrm{CBS}$ data. All energies are in $\mathrm{kcal} / \mathrm{mol}$.

\begin{tabular}{|c|c|c|c|c|}
\hline Structure & $\operatorname{CCSD}(\mathrm{T}) / \mathrm{CBS}^{a b}$ & AMOEBA $+{ }^{a c}$ & AMOEBA+(CF) ${ }^{a}$ & MB-UCB ${ }^{d}$ \\
\hline Smith01 & -4.97 & $-5.42(-4.96)$ & -4.98 & -5.15 \\
\hline Smith02 & -4.45 & $-4.57(-4.11)$ & -4.37 & -4.78 \\
\hline Smith03 & -4.42 & $-4.45(-4.00)$ & -4.29 & -3.86 \\
\hline Smith04 & -4.25 & $-5.20(-4.75)$ & -4.09 & -3.11 \\
\hline Smith05 & -4.00 & $-4.53(-4.08)$ & -3.53 & -3.68 \\
\hline Smith06 & -3.96 & $-4.36(-3.90)$ & -3.38 & -3.21 \\
\hline Smith07 & -3.26 & $-4.15(-3.69)$ & -3.19 & -2.93 \\
\hline Smith08 & -1.30 & $-1.85(-1.39)$ & -1.38 & -1.15 \\
\hline Smith09 & -3.05 & $-3.67(-3.22)$ & -3.03 & -2.99 \\
\hline Smith10 & -2.18 & $-2.79(-2.34)$ & -2.27 & -2.07 \\
\hline \multicolumn{2}{|r|}{ RMSE } & $0.59(0.28)$ & 0.25 & 0.51 \\
\hline
\end{tabular}

${ }^{a}$ BEs using MP2-optimized geometry for dimer and monomer; 31,38

${ }^{b} \mathrm{CCSD}(\mathrm{T}) / \mathrm{CBS}$ values were taken from reference; 40

${ }^{c}$. Values in paresntheses are BEs using MP2-optimized dimer and experimental monomer. These values were reported in the previous AMOEBA+ publication; 26

${ }^{d .}$ These values were taken from reference. 39 